\title{
A BIOMETRICAL ANALYSIS OF A MATING CHARACTERISTIC IN DROSOPHILIA
}

\author{
HELENA C. KRAEMER and SEYMOUR KESSLER \\ Department of Psychiatry, Stanford University, Stanford, California 94305
}

Received 8.ii.74

\section{SUMmary}

\begin{abstract}
A model is described for the biometrical analysis of interaction behavioural characteristics.

The model is illustrated by analysis of the mating speed of Drosophila pseudoobscura derived from crosses of fast and of slow mating strains generated through artificial selection.
\end{abstract}

\section{INTRODUCTION}

THE application of biometrical techniques to the analysis of behavioural aspects of the phenotype has blossomed in recent years; advances in this area are reviewed by Broadhurst (1960), Broadhurst and Jinks (1961), Roberts (1968) and by others. The biometrical approach is applicable in studies of quantitative inheritance when the characteristic of interest allows the measurement or scoring of individual organisms. However, when the character being studied produces a phenotypic value which is the simultaneous resultant of the interaction of two organisms, the usual biometrical analyses may no longer apply. Thus, in effect, mating and other important social behaviours would be excluded from the analytic armament offered by this statistical approach. The present study attempts to generalise biometrical techniques for use in the study of interaction behavioural characteristics, using as a model the mating behaviour of Drosophila.

\section{THEORY}

(i) Summary of the method of components of variance (Mather, 1949)

It is assumed that at each of the $K$ gene loci with two alleles, $A_{i}, a_{i}$, affecting the characteristic, the magnitude of the contribution to the genotypic value is given by:

$$
A_{i} A_{i}: d_{i} ; A_{i} a_{i}: h_{i}+d_{m i} ; a_{i} A_{i}: h_{i}-d_{m i} ; a_{i} a_{i}:-d_{i} .
$$

For $i=1,2, \ldots, K$, all $d_{i}$ have the same sign but no sign restriction is imposed on $h_{i}$ or $d_{m i}$. The true breeding strain $\mathrm{P}_{1}$ is defined to be that with $A_{i} A_{i}$ at location $i ; \mathrm{P}_{2}$ has $a_{i} a_{i} ; \mathrm{F}_{1 a}$ has $A_{i} a_{i}$ and $\mathrm{F}_{1 b}$ has $a_{i} A_{i}$.

Let

$$
[d]=\sum_{i=1}^{K} d_{i}, \quad[h]=\sum_{i=1}^{K} h_{i}, \quad\left[d_{m}\right]=\sum_{i=1}^{K} d_{m i} \text {. }
$$

The mid-parental value is $m$ and $\varepsilon$ denotes the environmental deviation, a standardised normal random variable assumed to be independent of the genetic contribution to the phenotypic value. Table 1 summarises this model. Table 2 summarises the statistics resulting from a proper scaling of 
the characteristic of the backcross and $\mathrm{F}_{2}$ strains derived from the true breeding strains, based on this model.

TABLE 1

Summary of model: true breeding strains

\begin{tabular}{|c|c|c|c|c|}
\hline Strain & $\begin{array}{l}\text { At gene } \\
\text { location }\end{array}$ & $\begin{array}{c}\text { Genotype } \\
\text { effect at }\end{array}$ & $\begin{array}{l}\text { Mean genotype } \\
\text { effect }\end{array}$ & Variance \\
\hline$P_{1}$ & $A_{i} A_{i}$ & $d_{i}$ & $m+[d]$ & $\sigma^{2}$ \\
\hline $\mathbf{P}_{2}$ & $a_{t} a_{i}$ & $-d_{i}$ & $m-[d]$ & $\sigma^{2}$ \\
\hline$F_{1 a}$ & $A_{i} a_{i}$ & $h_{i}+d_{m i}$ & $m+[h]+\left[d_{m}\right]$ & $\sigma^{2}$ \\
\hline$F_{1 b}$ & $a_{i} A_{i}$ & $h_{i}-d_{m i}$ & $m+[h]-\left[d_{m}\right]$ & $\sigma^{2}$ \\
\hline
\end{tabular}

TABLE 2

Summary of model: backcross and $F_{2}$ strains

\begin{tabular}{|c|c|c|c|}
\hline Strain & Parents* & $\begin{array}{l}\text { Mean genotypic } \\
\text { value }\end{array}$ & $\begin{array}{c}\text { Variance of } \\
\text { phenotypic values }\end{array}$ \\
\hline $\mathbf{B}_{1 a}$ & $\mathrm{P}_{1} \times \mathrm{F}_{1 a}$ or $\mathrm{P}_{1} \times \mathrm{F}_{1 b}$ & $m+\frac{1}{2}\left([d]+[h]+\left[d_{m}\right]\right)$ & $\frac{1}{4} \sum_{i}\left(d_{i}-h_{i}-d_{m i}\right)^{2}+\sigma^{2}$ \\
\hline $\mathbf{B}_{1 b}$ & $\mathrm{~F}_{1 a} \times \mathrm{P}_{1}$ or $\mathrm{F}_{1 b} \times \mathrm{P}_{1}$ & $m+\frac{1}{2}\left([d]+[h]-\left[d_{m}\right]\right)$ & $\frac{1}{4} \sum_{i}\left(d_{i}-h_{i}+d_{m i}\right)^{2}+\sigma^{2}$ \\
\hline $\mathbf{B}_{2 a}$ & $\mathbf{P}_{2} \times \mathbf{F}_{1 a}$ or $\mathbf{P}_{2} \times \mathbf{F}_{1 b}$ & $m+\frac{1}{2}\left(-[d]+[h]-\left[d_{m}\right]\right)$ & $\frac{1}{4} \sum_{i}\left(d_{i}+h_{i}-d_{m i}\right)^{2}+\sigma^{2}$ \\
\hline $\mathbf{B}_{2 b}$ & $\mathbf{F}_{1 a} \times \mathbf{P}_{2}$ or $\mathbf{F}_{1 b} \times \mathbf{P}_{2}$ & $m+\frac{1}{2}\left(-[d]+[h]+\left[d_{m}\right]\right)$ & $\frac{1}{4} \Sigma\left(d_{i}+h_{i}+d_{m i}\right)^{2}+\sigma^{2}$ \\
\hline$F_{2}$ & $\mathrm{~F}_{1}(a$ or $b) \times \mathrm{F}_{1}(a$ or $b)$ & $m+\frac{[h]}{2}$ & $\frac{1}{2} \Sigma d_{i}^{2}+\frac{1}{4} \Sigma h_{i}^{2}+\frac{1}{4} \Sigma d_{m i}^{2}+\sigma^{2}$ \\
\hline
\end{tabular}

* The convention that $P_{1} \times F_{1 a}$ is a cross between a $P_{1}$ female and a $F_{1 a}$ male is observed throughout this presentation.

Each of the genotype means of table 2 is the average of some set of genotype means of the non-segregating strains. Using the name of the strain to represent its genotype mean, we find:

$$
\begin{aligned}
& \mathrm{B}_{1 a}=\frac{1}{2}\left(\mathrm{P}_{1}+\mathrm{F}_{1 a}\right) \\
& \mathrm{B}_{1 b}=\frac{1}{2}\left(\mathrm{P}_{1}+\mathrm{F}_{1 b}\right) ; \\
& \mathrm{B}_{2 a}=\frac{1}{2}\left(\mathrm{P}_{2}+\mathrm{F}_{1 a}\right) ; \\
& \mathrm{P}_{2 b}=\frac{1}{2}\left(\mathrm{P}_{2}+\mathrm{F}_{1 b}\right) ; \\
& \mathrm{F}_{2}=\frac{1}{4}\left(\mathrm{P}_{1}+\mathrm{P}_{2}+\mathrm{F}_{1 a}+\mathrm{F}_{1 b}\right) .
\end{aligned}
$$

The degree to which experimental data conform to the requirements of these relationships provides a test of the adequacy of the scale of measurement used for the data (Mather, 1949). If these requirements are satisfied, algebraic manipulation of the variances of table 2 leads to estimation procedures for a number of genetic parameters.

\section{(ii) Mating characteristics}

Usually one observes a phenotypic value $P$ such that $P=G+\varepsilon$ where $G$ is the genetic contribution and $\varepsilon$ an environmental effect. In studying parameters of mating behaviour one can only observe a function of the 
phenotypic values of the male and female $P=f\left(G_{q}+\varepsilon_{\phi}, G_{\delta^{*}}+\varepsilon_{\delta^{*}}\right)$. In general, if the male and female of a given species exert equal control of the mating characteristics, $f$ is a function symmetric in its two arguments. If one sex completely dominates, $f$ is a function of a single one of its arguments. Usually, the functional form is not known and unless the function can be assumed to be linear in form, is not readily estimable from data. Therefore we seek a scaling of the observable material such that

$$
P^{\prime}=m+\alpha\left(G_{q}+\varepsilon_{q}\right)+\beta\left(G_{\sigma^{\prime}}+\varepsilon_{\delta^{\prime}}\right),
$$

where $\alpha$ and $\beta$ reflect differences in the sex drive of the participants in the mating.

For mating characteristics in a "true breeding strain" the observed values for the intrastrain matings remains stable under selection from generation to generation and

$$
E\left(P^{\prime}\right)=m+\alpha G_{\text {o }}+\beta G_{\sigma^{\prime}},
$$

$\operatorname{var}\left(P^{\prime}\right)=\left(\alpha^{2}+\beta^{2}\right) \sigma^{2}$, with $\sigma^{2}$ the population phenotype variance. In table 3 are computed the mean value of the mating characteristics for matings among the genotypes of table 1 .

Although there are ostensibly six unknown parameters $\left(m, \alpha, \beta,[d],[h],\left[d_{m}\right]\right)$, these are not uniquely defined, since for any constant, $c$, the set $(m, \alpha / c, \beta / c$, $\left.c[d], c[h], c\left[d_{m}\right]\right)$ yields exactly the same means. To set the scale and have a unique solution, $\alpha+\beta$ is arbitrarily set equal to 1 .

\section{(iii) Method of analysis}

Suppose $r$ matings $(r>1)$ of each of the 16 possible crosses among the non-segregating groups are observed, a single observation being denoted $y_{i j k}(i, j=1$ to $4, k=1$ to $r)$. A direct estimation procedure for the parameters $[d],[h],\left[d_{m}\right]$ and $\beta$ (or $\alpha$ ) can be derived, but a simpler approach evolves from the observation of the similarity in structure between this analysis and a $4 \times 4$ diallel analysis, the difference being, of course, that here the observation is on the mating itself while in the diallel cross it is on the progeny of the mating.

The first step in analysis is to note that each entry $\bar{y}_{i j}$. and its reciprocal cross are such that

$$
\begin{aligned}
& E\left(\bar{y}_{i j .}\right)=m+\alpha X+\beta Y, \\
& E\left(\bar{y}_{j i .}\right)=m+\alpha Y+\beta X,
\end{aligned}
$$

where $X$ and $Y$ are genotype means. Therefore with

$$
\begin{gathered}
a_{i j}=\frac{\bar{y}_{i j .}+\bar{y}_{j i .}}{2} \text { and } d_{i j}=\frac{\bar{y}_{i j .}-\bar{y}_{j i .}}{2} \\
E\left(a_{i j}\right)=m+\frac{X+Y}{2}, \\
E\left(d_{i j}\right)=(\alpha-\beta) \frac{(X-Y)}{2} .
\end{gathered}
$$

* The dot notation is used. Where a subscript is replaced by a dot, summation occurs over all values of that subscript. If, in addition, there is a bar the average is taken. Thus $y_{i j}$. is the sum of the $r$ observations in the $(i, j)$ th cell, and $\bar{y}_{i j}$. is the average of observations in that cell. 


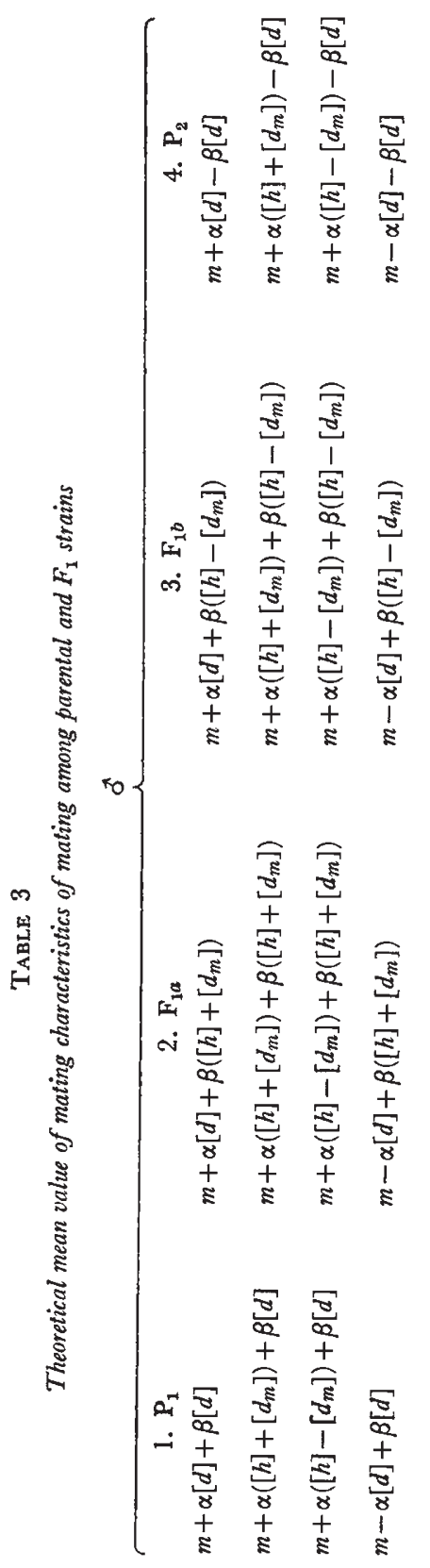

$$
\begin{aligned}
& \text { मा से से } \\
& \underbrace{-\dot{v} \dot{\circ}}
\end{aligned}
$$


The portion of the analysis model dealing with the averages, $a_{i j}$, is completely linear in the genotype parameters, that dealing with the differences, $d_{i j}$, depends heavily on the differential sex control parameter $(\alpha-\beta)$.

It is then straightforward to isolate the orthogonal linear contrasts related to $[d],[h]$ and $\left[d_{m}\right]$ from the analysis of the averages, and that related to $(\alpha-\beta)[d]$ (since $[d]$ is assumed non-zero) from the analysis of the differences. There are two residuals, each of which reflects different aspects of inadequacy of the linear model.

The complete analysis appears in table 4. The following notation is used:

$$
\begin{aligned}
a_{i j} & =\frac{1}{2}\left(\bar{y}_{i j .}+\bar{y}_{j i .}\right), \\
d_{i j} & =\frac{1}{2}\left(\bar{y}_{i j .}-\bar{y}_{j i .}\right), \\
R_{i} & =\bar{y}_{i . .}-\bar{y}_{\ldots} \ldots \text { (row or female mean deviation), } \\
C_{i} & =\bar{y}_{. i .}-\bar{y}_{\ldots} \ldots(\text { column or male mean deviation), } \\
{[\hat{d}] } & =\frac{1}{2}\left(R_{1}+C_{1}-R_{4}-C_{4}\right), \\
{[\hat{h}] } & =\left(R_{2}+C_{2}+R_{3}+C_{3}\right), \\
{\left[\hat{d}_{m}\right] } & =\frac{1}{2}\left(R_{2}+C_{2}-R_{3}-C_{3}\right), \\
\Delta & =\left(\widehat{\alpha-\beta)[d]}=\frac{1}{2}\left(R_{1}-C_{1}-R_{4}+C_{4}\right) .\right.
\end{aligned}
$$

TABLE 4

Theoretical analysis of variance

$\begin{array}{ccc}\text { Source of variation } & \text { d.f. } & \text { S.S. } \\ \text { Among cells } & 15 & r \Sigma\left(\bar{y}_{i j} .-\bar{y} \ldots\right)^{2} \\ \text { Among averages } & 9 & r \Sigma\left(a_{i j}-\bar{a}_{.} .\right)^{2} \\ {[d]} & 1 & 4 r\left[d^{2}\right)^{2} \\ {[h]} & 1 & 2 r[\hat{h}]^{2} \\ {\left[d_{m}\right]} & 1 & 4 r\left[\hat{d}_{m}\right]^{2} \\ \text { Residual } & 6 & * * \\ \text { Among differences } & 6 & r \sum d_{i j}{ }^{2} \\ (\alpha-\beta) \text { : Sex control } & 1 & 4 r \Delta^{2} \\ \text { Residual } & 5 & * \\ \text { Within cells = error } & 16(r-1) & \Sigma\left(y_{i j r}-\bar{y}_{i j} .\right)^{2} \\ \text { Total } & 16 r-1 & \Sigma\left(y_{i j r}-\bar{y}_{\ldots} \ldots\right)^{2}\end{array}$

* Obtained by subtraction.

\section{Materials AND METHODS}

A detailed description of the experimental materials and procedures may be found in Kessler (1968). Briefly, a heterogeneous wild-type population of Drosophila pseudoobscura was subjected to selection for fast and for slow mating speed. Flies derived from the 38th and 39th generations of selection were used in the present study. Twenty pairs of virgin females and males of each of the specified genotypes were placed together for 10 minutes. As matings occurred, the mated pairs were removed by means of an aspirator. The time at which each mating occurred and the number of pairs remaining 
unmated at the end of the observation period was recorded. Two observations of each type were made.

\section{Results}

A number of possible scoring systems were investigated, such as:

1. the percentage of maters and its arcsine transform;

2. $\left(\sum_{i=1}^{N} t_{i}+10 M\right) /(N+M)$ (where $t_{1}, t_{2}, \ldots, t_{N}$ are the mating times, $M$ the number of non-maters and $N$ the number of maters), its square, square root and reciprocal;

3. $\sum_{i=1}^{N} t_{i} / N$ with the score set at 10 if $N$ is zero.

The last measure was found to best satisfy the linearity requirement. Table 5 presents the means (each of two replicates) for this score. Thus $[\hat{d}]=-2.55,[\hat{h}]=-1.76,\left[\hat{d}_{m}\right]=-1.52, \hat{\alpha}=0.33, \hat{\beta}=0.67$. The analysis of variance shows that $[d],[h]$ and $\left[d_{m}\right]$ all differ significantly from

TABLE 5

Observed mean mating latency (in min.) between indicated genotypes averaged over two replicates

\begin{tabular}{|c|c|c|c|c|c|}
\hline \multirow{5}{*}{$\begin{array}{l}\text { F.F } \\
\text { F.S }\end{array}$} & F.F. & F.S. & S.F. & S.S. & $R_{i}$ \\
\hline & $2 \cdot 36$ & $2 \cdot 80$ & $6 \cdot 79$ & $7 \cdot 14$ & -0.34 \\
\hline & $3 \cdot 60$ & $2 \cdot 40$ & 3.96 & $7 \cdot 88$ & -0.65 \\
\hline & $4 \cdot 32$ & $3 \cdot 01$ & $6 \cdot 11$ & $5 \cdot 64$ & -0.34 \\
\hline & $4 \cdot 88$ & $5 \cdot 26$ & $7 \cdot 50$ & $8 \cdot 16$ & $1 \cdot 34$ \\
\hline$C_{i}$ & $-1 \cdot 32$ & -1.75 & +0.98 & $2 \cdot 09$ & \\
\hline
\end{tabular}

zero. The apparent greater control of the mating speed by the male is, however, not statistically significant. This result may be due to the lack of power of this test procedure based on only two replicates.

TABLE 6

Analysis of variance of observed data

\begin{tabular}{|c|c|c|c|c|}
\hline Source & d.f. & S.S. & M.S. & F \\
\hline Among cells & 15 & $119 \cdot 77$ & 7.98 & $2 \cdot 50^{*}$ \\
\hline Among averages & 9 & $96 \cdot 70$ & 10.74 & $3 \cdot 37 *$ \\
\hline$[d]$ & 1 & $52 \cdot 02$ & $52 \cdot 02$ & $23 \cdot 75^{* *}$ \\
\hline$[h]$ & 1 & $12 \cdot 39$ & $12 \cdot 39$ & $5 \cdot 66 * *$ \\
\hline$\left[d_{m}\right]$ & 1 & $18 \cdot 48$ & $18 \cdot 48$ & $8 \cdot 44 *$ \\
\hline Residual & 6 & $13 \cdot 81$ & $2 \cdot 30$ & n.s. \\
\hline Among differences & 6 & $23 \cdot 07$ & $3 \cdot 84$ & n.s. \\
\hline$(\alpha-\beta):$ Sex control & I & $6 \cdot 06$ & $6 \cdot 06$ & n.s. \\
\hline Residual & 5 & $17 \cdot 01$ & $3 \cdot 40$ & n.s. \\
\hline Within cells & 16 & 35.01 & $3 \cdot 19$ & \\
\hline Total & 31 & $154 \cdot 78$ & & \\
\hline
\end{tabular}


Since the two residuals are not significant, they may be pooled with Within Gells SS to yield a single estimate of $\sigma^{2}$ (from table 6):

$$
\left(\hat{\alpha}^{2}+\hat{\beta}^{2}\right) \hat{\sigma}^{2}: \frac{13 \cdot 81+17 \cdot 01+35 \cdot 01}{6+5+16}=2 \cdot 44,
$$

which leads to an estimate of the population phenotype variance $\sigma^{2}$ :

$$
\hat{\sigma}^{2}=4 \cdot 37 \text {. }
$$

These findings seem to differ from those previously reported (Kessler, 1968); however, the experimental protocol and the statistical model upon which the previous analysis was based differ considerably from that used in the present study.

\section{Analysis of bagkgross and $\mathrm{F}_{2}$ Generations}

The derivation of relationships analogous to those of table 2 is now straightforward. For example, for any member of the $\mathrm{B}_{1 a}$ strain the genotypic value is $\sum_{i=1}^{K} g_{i}$, where $g_{i}$ are independent random variables with $g_{i}=d_{i}+\varepsilon_{i}$ and to $h_{i}+m_{i}+\varepsilon_{i}$ each with probability $\frac{1}{2}$. Thus for a randomly mated female and male from this strain the measurement is

with mean

$$
m+\alpha \sum_{i=1}^{K} g_{i}+\beta \sum_{i=1}^{K} g_{i}^{\prime}+\alpha \varepsilon-\beta \varepsilon^{\prime}
$$

or

$$
m+\alpha\left([d]+[h]+\left[d_{m}\right]\right) / 2+\beta\left([d]+[h]+\left[d_{m}\right]\right) / 2
$$

$$
\frac{1}{2}(m+\alpha[d]+\beta[d])+\frac{1}{2}\left(m+\alpha\left([h]+\left[d_{m}\right]\right)+\beta\left([h]+\left[d_{m}\right]\right) .\right.
$$

Thus with the name of the strain to represent the mean intrastrain mating characteristic, i.e. the appropriate entries on the diagonal of the matrix of table $3: \mathrm{B}_{1 a}=\frac{1}{2}\left(\mathrm{P}_{1}+\mathrm{F}_{1 a}\right)$. In fact, all the usual scaling equations hold for a linear measure.

The variance of the intra- $\mathrm{B}_{1 a}$ matings is:

$$
\frac{\left(\alpha^{2}+\beta^{2}\right)}{4} \sum_{i=1}^{K}\left(d_{i}-h_{i}-d_{m i}\right)^{2}+\left(\alpha^{2}+\beta^{2}\right) \sigma^{2} .
$$

The variances of the intra-backcross and intra- $\mathrm{F}_{2}$ matings can all be shown to be the appropriate entry in table 2 multiplied by the factor $\left(\alpha^{2}+\beta^{2}\right)$. The estimators of $\alpha$ and $\beta$ can be used to adjust the variances if necessary to apply the usual techniques to estimate further genetic parameters.

If, on the other hand, mating is completely assortative, i.e. flies tend to mate with partners of their own genotypes, the means will be identical with the mean genotypic values of table 2 with variance in each case simply equal to $\sigma^{2}$.

In table 7 are the statistics from the matings among the progeny of the matings previously studied, with expectations based on a random mating model, and a completely assortative model. With the exception of $\mathrm{B}_{2 b}$, agreement between the observed means and those based on a random mating model is quite good. 
TABLE 7

Observed and predicted means of mating latency of backcross and $F_{2}$ generations

\begin{tabular}{|c|c|c|c|c|c|}
\hline Strain & $\begin{array}{l}\text { Number of } \\
\text { replicates }\end{array}$ & $\begin{array}{l}\text { Observed } \\
\text { mean }\end{array}$ & $\begin{array}{l}\text { Observed } \\
\text { variance }\end{array}$ & $\begin{array}{c}\text { Random } \\
\text { expection } \pm \text { s.d. }\end{array}$ & $\begin{array}{l}\text { Completely } \\
\text { assortative } \\
\text { expectation }\end{array}$ \\
\hline $\mathrm{B}_{1 a}$ & 4 & $2 \cdot 12$ & $0 \cdot 110$ & $2 \cdot 38 \pm 1 \cdot 32$ & 3.08 \\
\hline $\mathrm{B}_{1 b}$ & 4 & $2 \cdot 85$ & $1 \cdot 23$ & $4 \cdot 24 \pm 1 \cdot 32$ & $4 \cdot 60$ \\
\hline $\mathrm{B}_{2 a}$ & 4 & 5.01 & 0.738 & $5 \cdot 28 \pm 1 \cdot 32$ & $7 \cdot 14$ \\
\hline $\mathrm{B}_{2 b}$ & 4 & $3 \cdot 38 * *$ & $1 \cdot 60$ & $7 \cdot 13 \pm 1 \cdot 32$ & $5 \cdot 62$ \\
\hline$F_{2}$ & 8 & $4 \cdot 15$ & 0.982 & $4.76 \pm 0.33$ & $5 \cdot 11$ \\
\hline
\end{tabular}
estimate

With $\hat{\alpha}=0.33$ and $\hat{\beta}=0.67$, assuming the random mating model, we

$$
\begin{aligned}
\sum_{i=1}^{K} \hat{d}_{i}^{2} & =\frac{4 \operatorname{var}\left(\mathrm{F}_{2}\right)-\operatorname{var}\left(\mathrm{B}_{1 a}\right)-\operatorname{var}\left(\mathrm{B}_{1 b}\right)-\operatorname{var}\left(\mathrm{B}_{2 a}\right)-\operatorname{var}\left(\mathrm{B}_{2 b}\right)}{\hat{\alpha}^{2}+\hat{\beta}^{2}} \\
& =0.448 .
\end{aligned}
$$

Letting

$$
\begin{gathered}
\vec{d}=\frac{1}{K} \sum_{i=1}^{K} d_{i} \text { and } S_{d}^{2}=\frac{1}{K} \sum_{i=1}^{K}\left(d_{i}-\partial\right)^{2}, \\
\frac{|\hat{d}|}{\sum_{i=1}^{K} \hat{d}_{i}^{2}} \approx \frac{K}{1+S_{d}^{2} / \bar{d}} \leq K,
\end{gathered}
$$

we estimate that $K$, the number of gene locations involved, is at least 6 . The potence measure $[h] / 2[d]$ is estimated to be 0.345 , which indicates that the genes which determine fast mating speed are dominant to those determining slow mating speed.

\section{Discussion}

Since many significant behaviours in man and in other animal species involve interactions between two or more conspecifics, it is of interest to develop methods by which such behaviour may be analysed utilising biometrical techniques. In the present study, mating speed in Drosophila has been used as an example of an interaction characteristic. Other interaction characteristics are being explored by Hay (1972).

Genotypic contributions to the speed of mating have been demonstrated in several species of Drosophila (Kessler, 1968, and references therein) where it appears to comprise an important component of fitness (Spiess and Langer, 1964a, $b$; Fulker, 1966; Prakash, 1967). The nature of the genotypic variance underlying mating speed in $D$. melanogaster has been studied by Parsons (1964) and Fulker (1966) utilising a diallel design and in $D$. pseudoobscura by Kessler (1969) using selective breeding and classical genetic analysis.

The problem posed by the interaction of the two sexes in the measurement of mating speed in Drosophila has no easy solution. Mating behaviour in 
Drosophila is sexually dimorphic; the female appears to be relatively passive during the courtship, whereas, the male is clearly active, engaging in various behaviours which have been described in detail elsewhere (Spieth, 1968). It is generally agreed that mating in non-anaesthetised Drosophila flies requires the proper synchronisation of the behaviour of both sexes. Attempts have been made to study male courtship behaviour by using inanimate models (Milani, 1950; Brown, 1964) and etherised (Streisinger, 1948) or decapitated (Spieth, 1966) female partners. However, inferences drawn from nonnaturalistic simulation of the mating interaction may not reflect characteristics of the true interaction. Fulker (1966), on the other hand, approached the problem of sex interaction in the analysis of mating speed by focusing only on the contribution of the male. Each male was confined with a standard number of $D$. melanogaster females. It was thus hoped to present to each male a common, constant test situation such that differences in mating speed between males could be ascribed to the genotypic differences between them. This approach certainly has advantages over the methods used in other studies; however, it does not circumvent the fact that behaviourally, mating speed is an interaction characteristic and information is lost by focusing only on the contribution of one of the participants of the interaction.

Careful construction of specialised analyses highlighting the interactive aspects of certain behaviour may yield substantial reward in terms of information made available about behavioural interactions as they may occur in naturalistic settings. The present study is a limited one in terms of the full range of possible problems of studying interaction characteristics, since it is specifically directed only to the problem of mating interactions. However, this approach may serve as a prototype of analyses of interactive characteristics.

Acknowledgments.-This research was supported by grants MH 14363 and MH 8304 .

\section{REFERENCES}

BROADHURST, P. L. 1960. Experiments in psychogenetics: Applications of biometrical genetics to the inheritance of behaviour. In Experiments in Personality, vol. 1, ed. H. J. Eysenck, pp. 3-102. Routledge and Kegan Paul, London.

BROADHURST, P. L., AND JINKs, J. L. 1961. Biometrical genetics and behavior: Reanalysis of published data. Psychol. Bull., 58, 337-362.

BRown, R. G. B. 1964. Courtship behaviour in the Drosophila obscura group. I. Drosophila pseudoobscura. Behaviour, 23,61-106.

FULKeR, D. W. 1966. Mating speed in male Drosophila melanogaster: A psychogenetic analysis. Science, 153, 203-205.

HAY, D. A. 1972. Recognition by Drosophila melanogaster of individuals from other strains or cultures: Support for the role of olfactory cues in selective mating? Eoolution, 26, 171 176.

KEssLer, s. 1968. The genetics of Drosophila mating behaviour. I. Organization of mating speed in Drosophila pseudoobscura. Anim. Behav., 16, 485-491.

KESSLER, s. 1969. The genetics of Drosophila mating behaviour. II. The genetic architecture of mating speed in Drosophila pseudoobscura. Genetics, 62, 421-433.

MATHER, K. 1949. Biometrical Genetics. Methuen, London.

MILANI, R. 1950. Release of courtship display in subobscura males simulated with dummies. Drosophila Information Service, 24, 88.

parsons, P. A. 1964. A diallel cross for mating speeds in Drosophila melanogaster. Genetics, 35, 141-151.

PRAKAsH, s. 1967. Association between mating speed and fertility in Drosophila robusta. Genetics, 57, 655-663. 
ROBERTS, R. G. 1968. Some concepts and methods in quantitative genetics. In BehaviorGenetic Analysis, ed., J. Hirsch, pp. 214-257. McGraw-Hill, New York.

SPIESS, E. B., AND LANGER, B. 1964a. Mating speed control by gene arrangements in Drosophila pseudoobscura homokaryotypes. Proc. Nat. Acad. Sci., U.S.A., 51, 1015-1019.

SPIESS, E. B., AND LANGER, B. $1964 b$. Mating speed control by gene arrangement carriers in Drosophila persimilis. Evolution, 18, 430-444.

SPIETH, н. T. 1966. Drosophilid mating behavior: The behavior of decapitated females. Anim. Behav., 14, 226-235.

SPIETH, н. T. 1968. Evolutionary implications of sexual behavior in Drosophila. In Evolutionary Biology, vol. II, eds., Th. Dobzhansky, M. K. Hecht and W. C. Steere, pp. 157193. Appleton-Century-Crofts, New York.

STREISINGER, G. 1948. Experiments on sexual isolation in Drosophila. IX. Behavior of males with etherized females. Evolution, 2, 187-188. 